\title{
Proposta de sistemática para a integração da ergonomia no projeto de produtos
}

\author{
Systematic proposal for the integration of human factors \\ in product design
}

Fabiola Reinert ${ }^{1}$

Leila Amaral Gontijo² 


\section{Resumo}

O objetivo deste artigo é propor uma sistemática para a integração da ergonomia no projeto de produtos, com base na abordagem de projeto centrado no usuário. Sendo assim, é apresentada uma visão geral da sistemática, além da sugestão dos métodos e ferramentas de apoio para cada fase do processo de projeto. Ao final, é exposta uma avaliação da sistemática realizada por especialistas na área de projeto de produtos e ergonomia, sendo positivamente avaliada.

Palavras-chave: Desenvolvimento de Produtos; Metodologia de Projeto de Produtos; Ergonomia; Projeto Centrado no Usuário.

\section{Abstract}

The aim of this paper is to propose a systematic for the integration of human factors in product design, based on the user-centered design approach. Thus, an overview of the systematics is presented, as well as the suggestion of methods and support tools for each phase of the design process. At the end, an evaluation of the systematics carried out by specialists in the area of product design and human factors is presented, being evaluated positively.

Key-words: Product Development; Product Design Methodology; Human factors; User-Centered Design.

ISSN: 2316-7963

${ }^{1}$ Doutoranda em Engenharia de Produção, UFSC (fabiola.reinert@gmail.com) 2 Doutora em Ergonomia, UFSC 


\section{Introdução}

Inevitavelmente, todos já tiveram alguma dificuldade em interagir com um produto como, por exemplo, ao não conseguir abrir um frasco de vidro, mesmo utilizando uma grande força para fazer girar a tampa, ou ao empurrar uma porta quando esta deveria ser puxada. Situações como estas são resultados da falta de ergonomia, ou seja, a inadequação destes produtos às capacidades humanas e surgem quando estes não são corretamente pensados para a situação real de utilização no dia-a-dia e para o público que os vai utilizar (CRUZ, 2010).

De acordo com Merino (2014) o desenvolvimento de projetos com ênfase na ergonomia é uma necessidade natural, neste sentido as formas de projetar devem considerar as capacidades e limitações do ser humano. Entretanto, este enfoque se apresenta apenas na dimensão teórica, ou seja, é citado nos processos de desenvolvimento de projetos, mas na prática é negligenciado e até desconsiderado (MERINO, 2014). Para Mossink (1990), a incorporação da ergonomia ou fatores humanos nos processos de projeto depende, em grande parte, de um interesse pessoal do projetista.

Segundo a IEA (2017), a ergonomia (ou fatores humanos) é a disciplina científica relacionada à interação entre os seres humanos e outros elementos ou sistemas, que contribui para o planejamento, projeto e a avaliação de tarefas, postos de trabalho, produtos, ambientes e sistemas de modo a torná-los compatíveis com as necessidades, habilidades e limitações das pessoas.

Uma abordagem da ergonomia que vem ganhando espaço no desenvolvimento de produtos é o projeto centrado no usuário (User Centered Design - UCD), que, apesar de ter sido inicialmente aplicado no desenvolvimento de sistemas de computadores, tem gradualmente se infiltrado em outras disciplinas de projeto (KELLY, 2014).

Para Rubin e Chisnell (2008), o projeto centrado no usuário representa as técnicas, métodos e procedimentos para a concepção de produtos e sistemas utilizáveis, com a filosofia que coloca o usuário no centro do processo. Barrington (2007) afirma que o objetivo do UCD é projetar o produto para atender o usuário, em oposição ao projeto de produto centrado no objeto, no qual o foco está na solução técnica.

O UCD foi formalizado através da norma ISO 13407 - Human Centred Design Processes for Interactive Systems, de junho de 1999, posteriormente atualizada pela ISO 9241-210 (2010), que orienta a incorporação de atividades centradas no usuário para a obtenção de qualidade de uso em todo o ciclo de vida de sistemas interativos. A norma descreve o projeto centrado no usuário como uma atividade multidisciplinar, que incorpora conhecimento e técnicas de ergonomia com o objetivo de aumentar a eficácia e produtividade e melhorar as condições humanas de trabalho, além de ser "complementar às metodologias de projetos existentes e fornece uma perspectiva centrada no ser humano que pode ser integrada em diferentes processos de projeto e desenvolvimento" (ISO 9241-210, 2010, p. 8).

Para Neumann e Village (2012) é fundamental encontrar maneiras de integrar a ergonomia em cada estágio do desenvolvimento do projeto. Entretanto, uma abordagem para a incorporação da ergonomia ao projeto de produtos deveria penetrar na linguagem e nas técnicas comumente utilizadas pelos engenheiros (TOSSETO, 2013), utilizando assim uma metodologia de projeto já conhecida e estruturada. 
Nesse contexto, partindo-se dos pressupostos de que a ergonomia é pouco considerada na prática projetual da engenharia (REINERT; GONTIJO, 2017a), e que as metodologias de projeto de produto de engenharia não apresentam adequadamente a utilização da ergonomia (REINERT; GONTIJO, 2017b), pretendeu-se integrar a ergonomia em uma metodologia conceituada e validada, com o intuito de aumentar as chances do uso efetivo da abordagem ergonômica no desenvolvimento de produtos.

Considerando que, segundo Pheasant (1997), o projeto centrado no usuário se resume na abordagem ergonômica em relação ao projeto de produtos, acredita-se que uma abordagem formalizada e normatizada como o projeto centrado no usuário (ISO 9241-210) associado a uma metodologia bem estruturada no campo da engenharia, pode auxiliar os engenheiros a melhor compreender e aplicar a ergonomia no processo de desenvolvimento de produtos. Sendo assim, foi desenvolvida uma sistemática para integração da ergonomia no projeto de produtos, apresentada a seguir.

2. SISTEMÁTICA PARA INTEGRAÇÃO DA ERGONOMIA NO PROJETO DE PRODUTOS - SIEPP

Para o desenvolvimento da sistemática, foi considerado como base a metodologia de projeto de produtos de Back et al. (2008), por ser a mais utilizada pelos alunos de engenharia da Universidade Federal de Santa Catarina (REINERT; GONTIJO, 2017a), Universidade na qual a pesquisa foi desenvolvida. Sendo assim, foi feita a integração da ergonomia, por meio do projeto centrado no usuário, nesta metodologia, para que a sua utilização seja facilitada pelo conhecimento prévio dos alunos no processo.

A sugestão dos métodos e ferramentas de apoio a cada fase se dá pela relação dos mesmos com a fase do UCD e do PDP, de acordo com os estudos de Savi e Souza (2015), de Chaves, Bittercourt e Taralli (2013) e principalmente da própria norma ISO 9241-210 (2010) e da ISO/TR 16982 que apresenta os métodos que apoiam o projeto centrado no usuário.

A Figura 1 apresenta uma visão geral da sistemática, onde é evidenciada a correlação de cada fase de UCD com as fases do PDP, e os métodos que podem ser empregados, facilitando a visualização da integração da ergonomia no PDP. A visão detalhada de cada fase, bem como a integração das atividades de UCD e seus métodos, são apresentadas nos itens subsequentes. 
Figura 1 - Visão geral da sistemática.

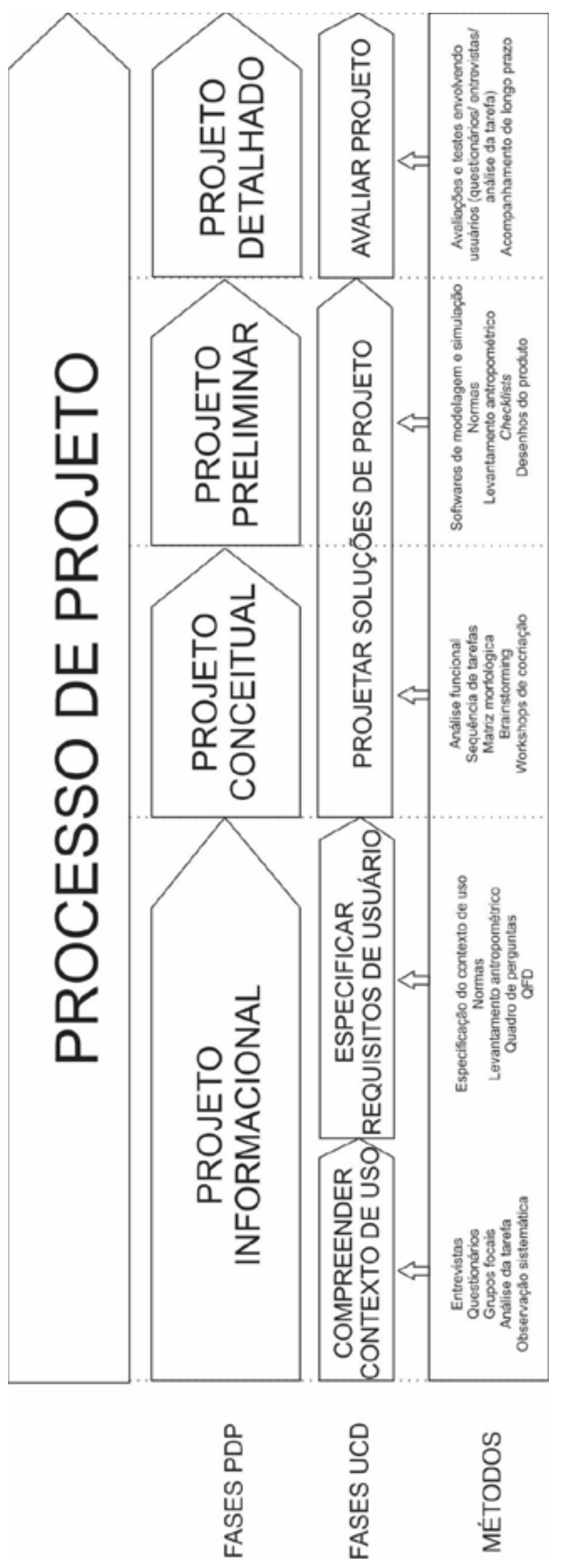




\subsection{FASE 1: PROJETO INFORMACIONAL}

As atividades relacionadas à integração da ergonomia na fase de projeto informacional são provenientes das etapas de compreender o contexto de uso e especificar requisitos de usuário do $U C D$, tendo como principais objetivos: descrever os usuários, suas características, objetivos e tarefas com o produto e ambiente de uso; e definir os requisitos de usuário em relação ao contexto de uso pretendido e aos objetivos do sistema, como mostra o quadro 1 a seguir.

Quadro 1 - Atividades, ferramentas e resultados das etapas 1 e 2 do UCD.

\begin{tabular}{|c|c|c|c|}
\hline ETAPAA & TIVIDADES & \multicolumn{2}{|c|}{\begin{tabular}{l|l} 
FERRAMENTASR & ESULTADOS
\end{tabular}} \\
\hline $\begin{array}{l}\text { COMPREENDER } \\
\text { O CONTEXTO } \\
\text { DE USO }\end{array}$ & $\begin{array}{l}\infty \quad \text { Coletar e analisar } \\
\text { informações sobre o } \\
\text { contexto de uso atual } \\
\infty \quad \text { Especificar o } \\
\text { contexto que será aplicado } \\
\text { ao futuro produto }\end{array}$ & $\begin{array}{l}\text {-Entrevistas } \\
\text {-Questionários } \\
\text {-Grupos focais } \\
\text {-Análise da tarefa } \\
\text {-Observação } \\
\text { sistemática }\end{array}$ & $\begin{array}{l}\text { Descrição dos usuários, } \\
\text { suas características, } \\
\text { objetivos e tarefas com o } \\
\text { produto e ambiente de } \\
\text { uso. }\end{array}$ \\
\hline $\begin{array}{l}\text { ESPECIFICAR } \\
\text { REQUISITOS DE } \\
\text { USUÁRIO }\end{array}$ & $\begin{array}{l}\infty \quad \text { Identificar } \\
\text { necessidades dos usuários } \\
\infty \quad \text { Derivar requisitos } \\
\text { de usuário } \\
\infty \quad \text { Solucionar } \\
\text { conflitos de escolha entre } \\
\text { os requisitos }\end{array}$ & $\begin{array}{l}\text {-Especificação do } \\
\text { contexto de uso } \\
\text {-Normas } \\
\text {-Levantamento } \\
\text { antropométrico } \\
\text {-QFD }\end{array}$ & $\begin{array}{l}\text { Declaração explicita dos } \\
\text { requisitos de usuário em } \\
\text { relação ao contexto de uso } \\
\text { pretendido e aos objetivos } \\
\text { do sistema. }\end{array}$ \\
\hline
\end{tabular}

A integração dessas atividades as atividades do projeto informacional foi feita considerando a correlação entre elas e a proximidade de objetivos. Sendo assim, são apresentadas na figura 2 as principais atividades do projeto informacional e onde as atividades do projeto centrado no usuário devem ser integradas, além dos métodos sugeridos para essas atividades.

De acordo com Back et al. (2008), a fase de projeto informacional se inicia com o plano do produto, proveniente da macrofase de planejamento. As principais etapas descritas pelos autores são: a apresentação do problema; a definição do ciclo de vida do produto e identificação dos usuários; a elicitação das necessidades dos usuários; a transformação das necessidades em requisitos de usuário; a conversão dos requisitos de usuário em requisitos de projeto; a análise de relacionamento entre requisitos e a definição das especificações de projeto (para maiores detalhamentos verificar a bibliografia).

$\mathrm{Na}$ atividade de "definição do ciclo de vida do produto e identificação dos usuários" é proposta a integração das atividades do UCD de "coletar e analisar as informações sobre o contexto de uso atual" e "especificar o contexto que será aplicado ao futuro produto". Essas atividades podem apoiar a definição do ciclo de vida e vice-versa. Para a realização dessas atividades, podem ser utilizadas entrevistas e questionários com os usuários do produto, aplicando perguntas fechadas ou abertas, formuladas com base nas áreas de interesse nas quais as informações precisam ser levantadas (ISO/TR 16982; SAVI; SOUZA, 2015; NIELSEN, 1993; STANTON et al., 2005; PREECE et al., 2002), grupos focais, que levantam informações sobre alguma 
questão em foco em uma reunião com usuários específicos (ISO/TR 16982; SAVI; SOUZA, 2015; NIELSEN, 1993; STANTON et al., 2005; TULLIS E ALBERT, 2008), observação sistemática do usuário no ambiente de uso, em que o usuário é observado de forma direta (presença do observador) ou indireta (por meio de filmagem) em seu ambiente natural, a fim de entender como os mesmos utilizam o produto, levantar necessidades e informações sobre o contexto de uso (ISO/TR 16982; SAVI; SOUZA, 2015; STANTON, 1998; STANTON et al., 2005) e análise da tarefa do usuário com o produto, que coleta detalhadamente informações sobre a realização da atividade, com o objetivo de compreender todas as tarefas que são feitas com o produto, registrando os movimentos, a sequência de tarefas, as maneiras diferentes de se utilizar o produto, percebendo falhas e problemas nessa interação (ISO/TR 16982; BAXTER, 1998; STANTON et al., 2005).

Figura 2 - Fase 1: Projeto informacional.

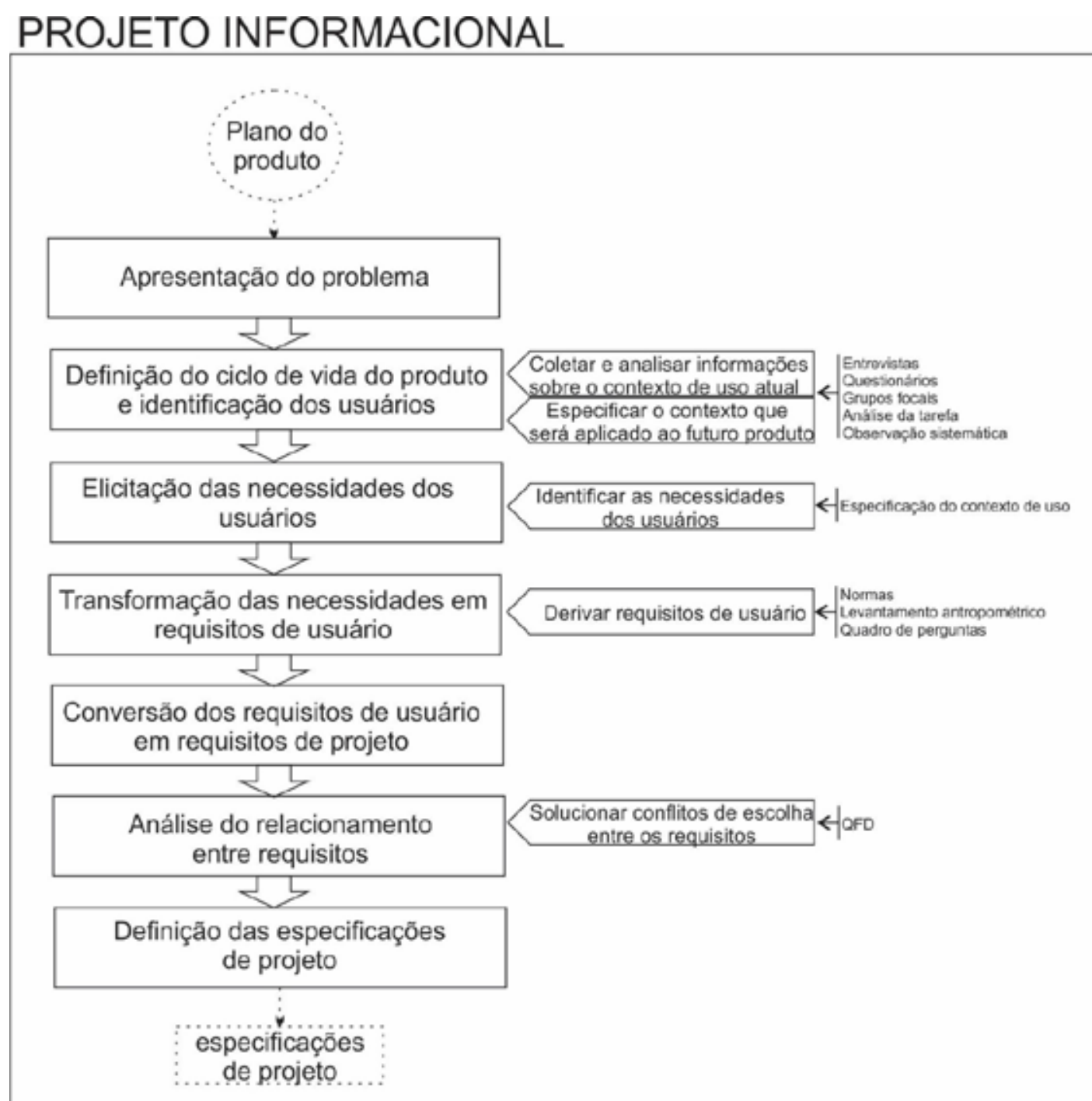

Segundo a norma ISO 9241-210(2010), o intuito dessas atividades e ferramentas é compreender o usuário, suas características, objetivos e tarefas com o produto, e o ambiente de uso do produto. Elas podem revelar necessidades, problemas e restrições que poderiam de outra forma passar despercebidas, mas que precisam ser abordadas pelo futuro produto. Ao final dessas atividades sugere-se organizar as 
informações obtidas para melhor utilizá-las nas atividades seguintes, por meio da descrição das informações como mostra o quadro 2 a seguir.

Quadro 2 - Modelo de especificação do contexto de uso.

Especificação do contexto de uso

Usuários: (quem é o público-alvo do produto)

Características dos usuários: (conhecimentos, habilidades, experiência, formação, a tributos físicos, hábitos, preferências)

Objetivos e tarefas dos usuários: (objetivos d o usuário com o produto, os o bjetivos g erais do sistema, q uais t arefas $\mathrm{s}$ ão e xecutadas com o produto, $\mathrm{d}$ e que maneira, $\mathrm{c}$ om qual frequência e

Ambientes de u so: (características r elevantes do a mbiente físico, social e cultural do u so do

$\mathrm{Na}$ atividade de "elicitação das necessidades dos usuários", deve-se integrar a atividade "identificar as necessidades dos usuários" do UCD, que tem como ferramenta de apoio a especificação do contexto de uso elaborada na atividade anterior. Nesse momento, as informações coletadas nas atividades anteriores são usadas para identificar as necessidades dos usuários com relação ao contexto.

A próxima atividade do UCD é "derivar os requisitos de usuário" que integra a atividade do projeto informacional "transformar as necessidades de usuário em requisitos de usuário". Nessa atividade podem ser utilizados normas e levantamento antropométrico para tornar as necessidades do usuário requisitos tangíveis e consistentes. No caso de usuários específicos, pode-se realizar o levantamento antropométrico junto aos próprios usuários, ou utilizar bibliografia renomada na área (TILLEY, ALVIN R. Henry Dreyfuss Associates. As medidas do homem e da mulher. Porto Alegre: Bookman, 2005).

Com relação às normas, o Código de Proteção e Defesa do Consumidor regulamenta como prática abusiva do fornecedor colocar no mercado de consumo qualquer produto ou serviço em desacordo com as normas expedidas pelos órgãos competentes, ou, se as normas específicas não existirem, pela Associação Brasileira de Normas Técnicas - ABNT (BRASIL, 1990). A ABNT elabora normas técnicas nos campos científicos, técnico, industrial, comercial e agrícola, além de incentivar o processo de normatização no país e promover o intercâmbio com entidades similares internacionais, como é o caso da Organização de Normalização Internacional - ISO. Indica-se o uso da NR17- Ergonomia da ABNT (BRASIL, 2002), além de normas da ISO, que geralmente contém itens que apresentam avanço em relação às melhores normas em vigor, provocando revisões nas normas nacionais (DIAS, 2000). Entretanto, normas internacionais tais como a DIN- Deustsches Institut für Normung (Alemanha) e AFNOR - Association Française de Normalization (França) também têm sua utilização recomendada.

Além disso, sugere-se o uso do quadro 3 abaixo, onde estão compiladas, a partir do tema de ergonomia, perguntas para apoio a definição de requisitos propostas por Carpes Jr. (2014). 
Quadro 3 - Quadro de perguntas para auxiliar na definição dos requisitos do usuário.

Qualquer pessoa poderá utilizar o produto? Que características ele possui para tornar isso possível? Como a equidade de utilização será garantida?

Quais serão as dimensões do corpo humano necessárias para o dimensionamento do produto? Os alcances estarão dentro da possibilidade do usuário? Qual o biotipo físico dos futuros usuários? Haverá conforto físico?

O produto será flexível no uso? Como isso será contemplado? Ele é adaptável às características dos usuários? É utilizável por destros e canhotos?

Quais serão as cargas e solicitações impostas pelo produto ao usuário?

O produto poderá ser de uso intuitivo? Como as pessoas de baixo nível de instrução poderão utilizálo? Que aspectos garantem sua simplicidade?

O produto se comunicará com o usuário? De que forma ele apresentará informações sobre seu estado ou desempenho? Haverá redundância na forma de comunicação?

Quais tipos de mostradores serão utilizados? Quais serão as chances de erro de leitura? Serão adequados ao ambiente? Serão adequados aos operadores? Em que número?

Quais tipos de comandos serão utilizados? Em que número?

Como será a iluminação no ambiente de uso? O produto terá iluminação própria? Haverá utilização de cores na iluminação? Quais serão utilizadas? Com qual objetivo?

O produto será tolerante com os erros de utilização? Como isso será contemplado? A falha segura está prevista? De que forma? Como erros de uso e utilização inadvertida serão suprimidos?

Qual será a duração do ciclo de operação do produto ou o tempo médio de utilização? Na operação, haverá excesso ou pouco estímulo? O sujeito estará sujeito à fadiga ou à monotonia? Haverá automação na operação?

Haverá ruídos? Deq ual frequência e intensidade? A que tipo de vibrações o usuário estará sujeito?

Qual será a postura física do operador durante o uso? Ela é confortável? Os esforços físicos e mentais serão aceitáveis? Os tempos de aplicação dos esforços são curtos? As ações repetitivas serão suprimidas? De que forma?

O tamanho do produto será adequado à operação? Que dimensões ele terá? Quais serão os valores máximos dos cursos dos comandos? Qual será a distância entre os mostradores e o usuário? Os mostradores serão legíveis? O produto permitirá a utilização por pessoas com deficiências? O produto poderá ser utilizado por pessoas com qualquer tamanho, formato ou aderência das mãos?

O produto estará sujeito a agentes externos agressivos, tais como chuva, umidade, frio, calor, poeira ou vibrações? De que forma essas condições ambientais poderão alterar a segurança do produto? Como o uso do produto poderá se tornar perigoso? Quais ações feitas pelo usuário poderão representar perigo? Poderão ocorrer situações perigosas durante a operação, a manutenção ou a limpeza? Quais características do produto serão inerentemente perigosas? Há eletricidade, lançamento de fragmentos, lâminas cortantes, ruídos ou outra característica perigosa no produto? Quais problemas de segurança o produto poderá oferecer? Como esses perigos podem ser evitados?

Quais subsistemas do produto poderão apresentar perigo? Qual é a probabilidade de perigos inerentes aos subsistemas provocares acidentes? A probabilidade de acidentes é pequena, média ou grande?H á perigo de choque elétrico, corte, morte ou outro?

Em caso de acidente, quais serão os possíveis danos, lesões ou prejuízos financeiros? Eles poderão ser quantificados? Existem normas aplicáveis em relação à segurança? Quais são? Quais são as recomendações em relação a esses produtos?

Quais diretrizes serão estabelecidas para melhorar a segurança dos produtos? Os perigos são elimináveis ou inerentes ao funcionamento do produto? É possível produzir um produto livre de características inseguras ou perigos? Se a resposta à pergunta anterior for negativa, é plausível construir barreiras e proteções para resguardar os usuários? Se isso também não for possível, é plausível prover o usuário com instruções e avisos adequados? Como se poderá limitar as consequências dos acidentes em relação à morte ou à lesão de pessoas, animais ou plantas, a danos em propriedades e no meio ambiente? Quais serão as necessidades de segurança para o produto, em termos de eliminação de perigos, isolamento de riscos (proteções), utilização de avisos?

Fonte: adaptado de Carpes Jr, 2014. 
Após essa atividade é feita a "conversão dos requisitos de usuário em requisitos de projeto" e então a "análise do relacionamento entre requisitos", onde a atividade "solucionar conflitos de escolha entre requisitos" é integrada. Para realizar essa atividade, pode-se fazer uso do método QFD, comumente utilizado na engenharia (BACK et al. 2008, ROZENFELD et al., 2006). O QFD se trata de um método de gestão do desenvolvimento de produtos que auxilia nesta etapa da sistemática, apesar de não fazer parte do escopo de ferramentas para a integração da ergonomia. Com o auxílio deste método, é possível correlacionar os requisitos do usuário com os requisitos do produto, definindo grau de importância e obtendo características mensuráveis dos mesmos. Com o grau de importância definido, pode-se classificar e hierarquizar os requisitos, solucionando conflitos de escolha entre requisitos.

Ao final do projeto informacional são definidas as especificações de projeto e passa-se para a fase seguinte.

\subsection{FASE 2: PROJETO CONCEITUAL}

A atividade relacionada à integração da ergonomia na fase de projeto conceitual é proveniente da etapa de projetar soluções de projeto do UCD, tendo como principal objetivo definir a ideia do produto levando em conta os requisitos de usuário, como mostra o quadro 4 a seguir.

\begin{tabular}{|c|c|c|c|}
\hline ETAPAA & TIVIDADES & FERRAMENTASR & ESULTADOS \\
\hline $\begin{array}{l}\text { PROJETAR } \\
\text { SOLUÇÕES DE } \\
\text { PROJETO }\end{array}$ & $\begin{array}{l}\infty \quad \text { Projetar tarefas } \\
\text { do usuário, interação do } \\
\text { usuário com o produto e } \\
\text { interface }\end{array}$ & $\begin{array}{l}\text {-Sequência de } \\
\text { tarefas( HTA) } \\
\text {-Workshops de } \\
\text { cocriação } \\
\text {-Brainstorming }\end{array}$ & $\begin{array}{l}\text { Ideia do produto definida } \\
\text { levando em conta os } \\
\text { requisitos de usuário. }\end{array}$ \\
\hline
\end{tabular}

$\mathrm{Na}$ figura 3, podem ser vistas as principais atividades do projeto conceitual e a integração da atividade do projeto centrado no usuário, além dos métodos de apoio sugeridos. A entrada desta fase são as especificações de projeto resultantes da fase de projeto informacional, e, segundo Back et al. (2008), o projeto conceitual tem como principais atividades: a geração de soluções de projeto e a seleção da concepção (para maiores detalhamentos verificar a bibliografia).

Figura 3 - Fase 2: Projeto conceitual.

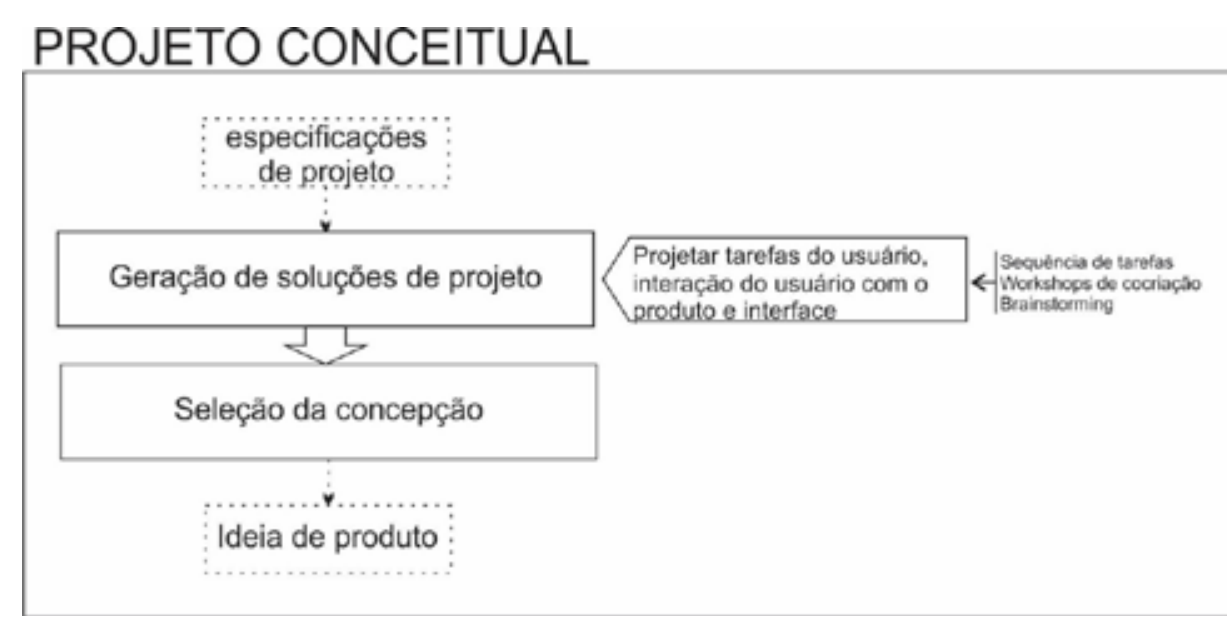


$\mathrm{Na}$ atividade de "geração de soluções de projeto" é proposta a integração da atividade do UCD de "projetar tarefas do usuário, interação do usuário com o produto e interface". Essa atividade auxilia a geração de ideias de produtos mais ergonômicas, decidindo como os usuários irão realizar as tarefas utilizando o produto, e permite a divisão de tarefas do sistema entre aquelas realizadas por seres humanos e aquelas realizadas pela tecnologia (ISO 9241-210:2010). Levando em conta o uso amplo na engenharia do método de análise funcional para dividir o produto em suas principais funções (que seriam as tarefas realizadas pela tecnologia) e o método de matriz morfológica, que apoia o encontro de soluções para essas funções (BACK et al., 2008; ROZENFELD et al. 2008), sugere-se juntamente com a análise funcional desenvolver a sequência de tarefas (HTA - Hierarchical Task Analysis) (ISO/TR 16982; STANTON, 1998), que dividirá as tarefas realizadas pelo usuário com o produto e permitirá utilizar também a matriz morfológica para encontrar soluções para essas tarefas. Segundo Stanton (1998), a sequência de tarefas fornece uma compreensão das tarefas que os usuários precisam executar para atingir determinados objetivos, sendo que essas tarefas podem ser quebradas em vários níveis de sub-tarefas. É usada para descrever as interações entre um usuário e um produto e permite explorar diversas abordagens possíveis para completar a mesma tarefa.

Além dos métodos sistemáticos de criatividade, mais comumente utilizados na engenharia (BACK et al.,2008), como a matriz morfológica, pode-se fazer uso de métodos intuitivos de criatividade, como o brainstorming (SAVI; SOUZA, 2015; STANTON, 1998; BAXTER, 1998) e workshops de cocriação, em que a geração de ideias é feira em conjunto com os usuários do produto (ISO/TR 16982; SAVI; SOUZA, 2015; NIELSEN, 1993; KARWOWSKI; SOARES; STANTON, 2011)

Após essa atividade, o projeto conceitual segue para a "seleção da concepção", que pode ser feita com diversos métodos de triagem, resultando em uma ideia de produto.

\subsection{FASE 3: PROJETO PRELIMINAR}

As atividades relacionadas à integração da ergonomia na fase de projeto preliminar são ainda provenientes da etapa de projetar soluções de projeto do UCD, assim como na fase de projeto conceitual, entretanto essas outras atividades tem como principal objetivo definir o leiaute do produto, como mostra o quadro 5 a seguir.

Quadro 5 - Atividades, ferramentas e resultados da etapa 3 do UCD.

\begin{tabular}{|l|l|l|l|}
\hline ETAPAA & \multicolumn{1}{|c|}{ TIVIDADES } & FERRAMENTASR & \multicolumn{1}{|c|}{ ESULTADOS } \\
\hline PROJETAR & $\infty \quad$ Tornar solução & -Softwares de & Leiaute do produto \\
SOLUÇÕES DE & mais concreta & modelagem e simulação & $\begin{array}{l}\text { especificado e } \\
\text { projetado. }\end{array}$ \\
PROJETO & $\infty \quad$ Alterar solução & -Normas & \\
& de projeto com base na & -Levantamento & \\
& avaliação centrada no & antropométrico & \\
& usuário & -Checklist & \\
& $\infty \quad$ Comunicar & -Desenhos do produto & \\
& solução de projeto & & \\
\hline
\end{tabular}


São apresentadas na figura 4 as principais atividades do projeto detalhado e onde as atividades do projeto centrado no usuário devem ser integradas, além dos métodos sugeridos para essas atividades.

De acordo com Back et al. (2008), a fase de projeto preliminar tem como entrada a ideia de produto, proveniente do projeto conceitual. As principais etapas descritas pelos autores são: modelagem e dimensionamento; simulação e otimização; análise de sensibilidade, compatibilidade e estabilidade; e especificação do produto e seus componentes (para maiores detalhamentos verificar a bibliografia).

Figura 4 - Fase 3: Projeto preliminar.

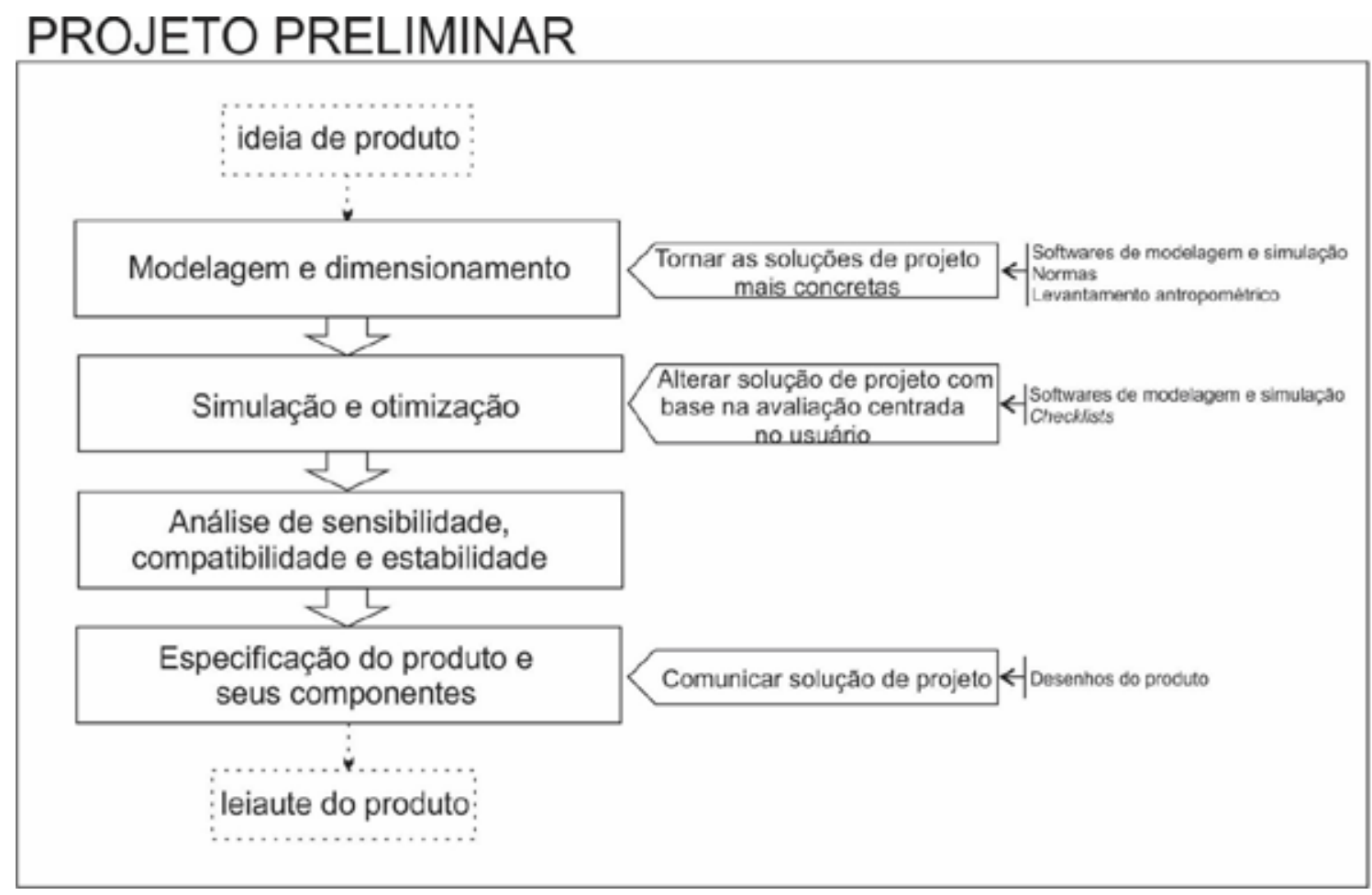

$\mathrm{Na}$ atividade de "modelagem e dimensionamento" é proposta a integração da atividade do UCD de "tornar as soluções de projeto mais concretas", com o apoio de softwares de modelagem e simulação, além das normas e levantamento antropométrico novamente, para auxiliar no dimensionamento.

Já na atividade de "simulação e otimização", a atividade de UCD a ser integrada é a de "alterar solução de projeto com base na avaliação centrada no usuário", que apoia o processo de otimização do produto. Sendo assim, podem ser usados softwares de modelagem e simulação e um Checklist de atendimento aos requisitos de usuário (ISO/TR 16982; STANTON, 1998), a fim de identificar alguma falha no atendimento dos requisitos e otimizar o projeto se necessário.

Na última atividade, "especificação do produto e seus componentes", é integrada a atividade de "comunicar solução de projeto", que tem mesma finalidade que a atividade do PDP. Nessa atividade devem-se realizar os desenhos do produto que permitam uma melhor comunicação da solução de projeto aos responsáveis pela execução. Ao final, tem-se como saída da fase o leiaute especificado do produto. 


\subsection{FASE 4: PROJETO DETALHADO}

A atividade relacionada à integração da ergonomia na fase de projeto detalhado é proveniente da etapa avaliar produto do UCD e tem como principal objetivo coletar feedback sobre os pontos fortes e fracos do produto sob a perspectiva do usuário, como mostra o quadro 6. Na figura 5 podem ser vistas as principais atividades do projeto detalhado e a integração da atividade de projeto centrado no usuário, além dos métodos de apoio sugeridos.

Quadro 6 - Atividades, ferramentas e resultados da etapa 4 do UCD.

\begin{tabular}{|c|c|c|c|}
\hline ETAPAA & TIVIDADES & FERRAMENTASR & ESULTADOS \\
\hline $\begin{array}{l}\text { AVALIAR } \\
\text { PRODUTO }\end{array}$ & $\begin{array}{l}\infty \quad \text { Avaliar se as } \\
\text { necessidades dos } \\
\text { usuários em relação ao } \\
\text { produto desenvolvido } \\
\text { foram atendidas }\end{array}$ & $\begin{array}{l}\text {-Avaliações e testes } \\
\text { envolvendo usuários } \\
\text { (questionários/ } \\
\text { entrevistas/ análise da } \\
\text { tarefa) } \\
\text {-Acompanhamento de } \\
\text { longo prazo }\end{array}$ & $\begin{array}{l}\text { Feedback sobre pontos } \\
\text { fortes e fracos do } \\
\text { produto sob a } \\
\text { perspectiva do usuário. }\end{array}$ \\
\hline
\end{tabular}

Figura 5 - Fase 4: Projeto detalhado.

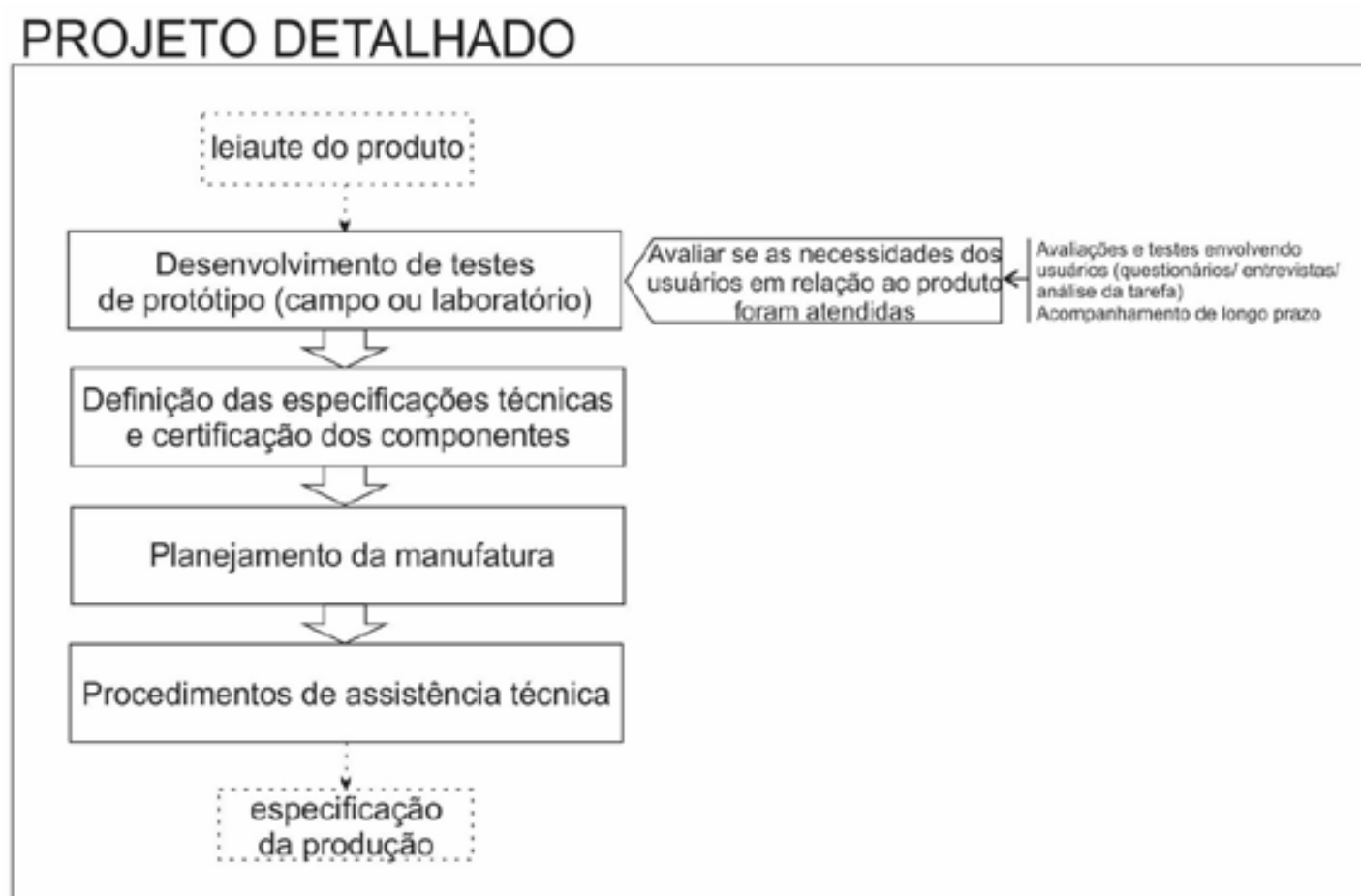

A entrada desta fase de projeto detalhado é o leiaute do produto, resultante do projeto preliminar. Back et al. (2008), apresentam como principais atividades do projeto detalhado: os testes de protótipo; a definição das especificações técnicas e certificação dos componentes; o planejamento da manufatura; e os procedimentos de assistência técnica (para maiores detalhamentos verificar a bibliografia).

$\mathrm{Na}$ atividade de "desenvolvimento de testes de protótipo (campo ou laboratório)" é proposta a integração da atividade do UCD de "avaliar se as necessidades dos usuários em relação ao produto foram atendidas". Essa atividade auxiliará os testes de 
protótipo, avaliando-o sob a perspectiva do usuário. Para a realização dessa atividade, podem ser utilizadas avaliações e testes envolvendo usuários, através de entrevistas, questionários, grupos focais e análise da tarefa (ISO/TR 16982; SAVI; SOUZA, 2015; STANTON et al., 2005), a fim de identificar junto aos usuários as possíveis mudanças que poderiam ocorrer para a melhoria do produto, além de permitir a coleta de informações sobre novas necessidades dos usuários, fornecer feedback sobre pontos fortes e fracos da solução de projeto e estabelecer baselines ou fazer comparações entre projetos. Ainda, pode ser realizado um acompanhamento de longo prazo do usuário com o produto, que permite feedbacks mais detalhados (ISO 9241-210:2010).

Após essa atividade, o projeto detalhado segue com as outras atividades resultando em uma especificação da produção.

\section{AVALIAÇÃO DA SISTEMÁTICA POR ESPECIALISTAS}

Para a avaliação da sistemática por especialistas, foi elaborado um formulário de avaliação com oito perguntas sobre a sistemática, baseadas nos critérios apresentados por Romano (2003), Montanha Jr. (2004), Leonel (2006) e Ibarra (2007) para avaliação de modelos de referência. Os critérios são com relação à aplicabilidade, clareza e contribuição da sistemática.

Foram contatados 15 especialistas, professores universitários e profissionais com conhecimento e experiência no processo de desenvolvimento de produtos de engenharia e/ou ergonomia. Foi enviada aos especialistas via correio eletrônico uma cópia da sistemática apresentando todas as fases, atividades e ferramentas relacionadas, e um formulário de avaliação a ser preenchido. Destes, 5 responderam no período de avaliação. O perfil dos especialistas que participaram desta avaliação é apresentado no quadro 7.

Quadro 7 - Perfil dos especialistas.

\begin{tabular}{|l|l|}
\hline Avaliador & Perfil do avaliador \\
\hline Especialista A & $\begin{array}{l}\text { Professora adjunta na área de Engenharia do Produto no departamento de } \\
\text { Engenharia de Produção da Universidade Federal de Santa Catarina, } \\
\text { ministrando a disciplina de projeto de produtos desde 2015. Doutora em } \\
\text { Engenharia de Produção e Mestre em Engenharia Mecânica pela UFSC. }\end{array}$ \\
\hline Especialista B & $\begin{array}{l}\text { Professor adjunto do Centro de Engenharias da Universidade Federal de } \\
\text { Santa Catarina, ministrando a disciplina de metodologia de projeto de } \\
\text { produtos desde 2010. Atua nas áreas de Gestão do Processo de } \\
\text { Desenvolvimento Integrado de Produtos, Gestão da Inovação e } \\
\text { Empreendedorismo. Mestre e Doutor em Engenharia Mecânica pela UFSC } \\
\text { na área de Projeto de Produtos. Pós-doutor em Engenharia de Produção } \\
\text { pela Escola de Engenharia da USP. }\end{array}$ \\
\hline Especialista C & $\begin{array}{l}\text { Professor adjunto do departamento de Engenharia de Produção e do } \\
\text { Programa de Pós Graduação em Engenharia de Produçãod a Universidade } \\
\text { Federal de Santa Catarina, ministrando disciplinas de metodologia de } \\
\text { projeto e desenvolvimento de produtos desde 2015. Atua na área de gestão } \\
\text { de projeto de produtos, desenvolvimento lean, plataforma de produtos e } \\
\text { customização em massa. Mestre e Doutor em Engenharia de Produção pela } \\
\text { UFRGS na área de Engenharia do Produto. }\end{array}$ \\
\hline
\end{tabular}




\begin{tabular}{|l|l|}
\hline Especialista D & $\begin{array}{l}\text { Professora associada do departamento de Engenharia de Produção e do } \\
\text { Programa de Pós Graduação em Engenharia de Produção da Universidade } \\
\\
\text { Federal de Santa Catarina. Atua na área de Ergonomia desde 2001, } \\
\text { lecionando em cursos de Engenharia de Produção, Design Industrial, } \\
\text { Arquitetura e Urbanismo, Engenharia de Materiais, Especialização em } \\
\\
\text { Engenharia e Segurança do trabalho e arquitetura de interiores. Mestre e } \\
\text { Doutora em Engenharia de Produção pela UFSC na área de Ergonomia. }\end{array}$ \\
\hline Especialista E & $\begin{array}{l}\text { Professora titular da Universidade Federal do Paraná. Mestre em } \\
\text { Engenharia de Produção pela UFSC na área de Ergonomia e Doutora em } \\
\text { Engenharia Mecânica pelo ITA na área de Engenharia de Produto. }\end{array}$ \\
\hline
\end{tabular}

Além do perfil apresentado acima, que já permite a visualização da área de atuação de cada especialista, foi desenvolvido um quadro para ilustrar o nível de conhecimento dos especialistas com relação às áreas de ergonomia e projeto de produtos, em escala likert. $O$ quadro 8 apresenta o número de respondentes para cada nível de conhecimento.

Quadro 8 - Conhecimento em Ergonomia e em Projeto de Produtos.

\begin{tabular}{|l|l|l|l|l|l|}
\hline & Nenhum & Pouco & Razoável & Bom & Muito \\
\hline Conhecimento em ergonomia & & 2 & 1 & & 2 \\
\hline $\begin{array}{l}\text { Conhecimento em projeto de produtos de } \\
\text { engenharia }\end{array}$ & & & & 2 & 3 \\
\hline
\end{tabular}

Os resultados obtidos nessa avaliação são apresentados no quadro 9, que mostra as perguntas e o número de respondentes em cada nível de atendimento do critério considerado. Serão considerados bons resultados as respostas de nível "bom" e "muito", pois sugerem que a sistemática atende totalmente, ou em muitos aspectos, o critério questionado.

Quadro 9 - Avaliação da sistemática quanto à aplicabilidade, clareza e contribuição.

\begin{tabular}{|c|l|l|l|l|l|}
\hline Questões & Não & Pouco & Razoavelmente & Bom & Muito \\
\hline $\begin{array}{l}\text { 1. A sistemática proposta tem } \\
\text { potencial para ser efetivamente } \\
\text { assimilada e aplicada? }\end{array}$ & & & & 1 & 4 \\
\hline $\begin{array}{l}\text { 2. A apresentação da sistemática e o } \\
\text { entendimento do processo são } \\
\text { compreensíveis? }\end{array}$ & & & 1 & 1 & 3 \\
\hline $\begin{array}{l}\text { 3. forma de integração e as } \\
\text { ferramentas propostas para o } \\
\text { projeto informacional são } \\
\text { adequadas? }\end{array}$ & & 1 & 1 & 2 & 2 \\
\hline $\begin{array}{l}\text { 4 forma de integração e as } \\
\text { ferramentas propostas para o } \\
\text { projeto conceitual são adequadas? }\end{array}$ & & & 1 & 2 & 2 \\
\hline $\begin{array}{l}\text { A forma de integração e as } \\
\text { ferramentas propostas para o } \\
\text { projeto preliminar são } \\
\text { adequadas? }\end{array}$ & & & 2 & 2 \\
\hline
\end{tabular}




\begin{tabular}{|c|c|c|c|}
\hline $\begin{array}{l}\text { 6. A forma de integração e as } \\
\text { ferramentas propostas para o } \\
\text { projeto detalhado são adequadas? }\end{array}$ & 1 & 1 & 3 \\
\hline $\begin{array}{l}\text { 7. A sequência de atividades do } \\
\text { UCD, na forma proposta, é } \\
\text { coerente com o PDP e permite o } \\
\text { andamento do processo? }\end{array}$ & & 3 & 2 \\
\hline $\begin{array}{l}\text { 8. A sistemática proposta apresenta } \\
\text { elementos que possibilitam a } \\
\text { integração da ergonomia no } \\
\text { processo de desenvolvimento de } \\
\text { produtos? }\end{array}$ & & 2 & 3 \\
\hline
\end{tabular}

Com base nos dados da tabela, pode-se perceber que a média geral das questões 1, 7 e 8 foi muito boa, o que significa que a sistemática tem potencial para ser efetivamente assimilada e aplicada, a sequência de atividades do UCD, na forma proposta, é coerente com o PDP e permite o andamento do processo, além de que a sistemática apresenta elementos que possibilitam a integração da ergonomia no processo de desenvolvimento de produtos.

As questões de 2 a 6, referentes à apresentação e compreensão da sistemática, e adequação das ferramentas propostas em cada fase, foram avaliadas positivamente pela maioria dos participantes, visto que as respostas se concentraram no nível "bom" e "muito". Pressupõe-se que a razão da nota "razoavelmente" nas questões de 2 a 6 por um dos respondentes se deva ao fato de que o documento entregue aos especialistas sobre a sistemática não era amplo e extenso a ponto de clarificar a forma de utilização de cada uma das ferramentas propostas, por ser um documento resumido, o que, segundo o especialista, poderia dificultar o entendimento de como realizar as atividades pelo leitor.

Sendo assim, a sistemática foi considerada satisfatória, visto que as respostas se concentraram nos níveis "bom" e "muito", indicativos da aceitação da sistemática pelos especialistas e do atendimento aos critérios estabelecidos no questionário.

\section{CONCLUSÃO}

Os estudos apresentados na introdução deste artigo evidenciam a importância da ergonomia no projeto de produtos e também a sua pouca utilização na prática projetual da engenharia, sendo essencial a sua integração em uma metodologia conceituada e validada para que suas chances de uso efetivo sejam aumentadas. $O$ conceito de projeto centrado no usuário como uma atividade multidisciplinar, que incorpora conhecimento e técnicas de ergonomia e fornece uma perspectiva centrada no ser humano que pode ser integrada em diferentes processos de projeto, auxilia essa integração de forma inovadora e eficiente.

A sistemática desenvolvida, ao relacionar as fases do projeto centrado no usuário da norma ISO 9241-210 (2010) com as fases do PDP proposto por Back et al. (2008), sugere os métodos e ferramentas que podem ser utilizados em cada fase do PDP com base no UCD, permitindo assim a integração da ergonomia no projeto de produtos. Tanto o fato de ser baseada em uma norma regulamentada, como o fato 
de ser desenvolvida sobre a metodologia de projeto de produtos de Back et al. (2008) tornam a sistemática mais consistente e passível de utilização por engenheiros e alunos de engenharia, pelo conhecimento prévio no PDP e facilidade de entendimento e busca de informações sobre a norma.

A avaliação por especialistas foi fundamental para verificar a aplicabilidade da sistemática proposta, além da sua clareza e contribuição, entretanto, é uma avaliação inicial que não possibilita analisar sua real utilização, sendo necessário, portanto, um estudo mais abrangente que verifique a real aplicabilidade prática da sistemática, a partir de um experimento com sua utilização. Dessa forma, será possível obter conclusões mais detalhadas da utilização da sistemática e integração efetiva da ergonomia no projeto de produtos de engenharia.

Sendo assim, espera-se que esta pesquisa inicial tenha tornado evidente a necessidade de maiores estudos na área, permitindo a análise de como a sistemática SIEPP pode auxiliar na integração da ergonomia no projeto de produtos.

\section{REFERÊNCIAS}

BACK, N. et al. Projeto integrado de produtos. Barueri - São Paulo: Manole, 2008.

BARRINGTON, S. Usability in the Lab: Techniques for Creat-ing Usable Products. Journal of the Association for Laboratory Automation, p. 6-11, 2007.

BAXTER, M. Projeto de Produto: guia prático para o desenvolvimento de produtos. São Paulo: Blücher, 1998.

BRASIL. Lei 8.078 de 11/09/90. Código de defesa do consumidor. Brasília, Diário Oficial da União, 1990. Disponivel em: <http://www.planalto.gov.br/ccivil_03/leis/ L8078compilado.htm>. Acesso em: 02 ago 2016.

BRASIL. Ministério do Trabalho (MT). Secretaria de inspeção do trabalho (SIT). Manual de aplicação da norma regulamentadora n17. 2 ed. Brasília: TEM, SIT, 2002. Disponível em: <http://www.mte.gov.br/seg_sau/pub_cne_manual_nr17.pdf>. Acesso em: 20 abr. 2015.

CARPES JR, W. Introdução ao projeto de produtos. Porto Alegre: Bookman, 2014.

CHAVES, I. G.; BITTENCOURT, J. P.; TARALLI, C. H. O design centrado no humano na atual pesquisa brasileira - uma análise através das perspectivas de klaus krippendorff e da ideo. Holos, ano 29, v.6, p. 213-225. 2013.

CRUZ, V. C. D. S. Design inclusivo: projeto para o desenvolvimento de uma ajuda técnica numa perspectiva de design inclusivo. Covilhã - Portugal, 2010. Dissertação (Mestrado) - Departamento de Engenharia Eletromecânica, Universidade da Beira Interior. 
DIAS, J. L. P. A. Integração da concepção ergonômica de maquinas e equipamentos na metodologia de projeto de produtos. Florianópolis, 2000. Dissertação (mestrado) - Departamento de Engenharia de Produção, Universidade Federal de Santa Catarina.

IBARRA, C. J. Sistematização do processo de mapeamento tecnológico de produtos. Florianópolis, 2007. Dissertação (mestrado) - Departamento de Engenharia Mecânica, Universidade Federal de Santa Catarina.

IEA (International Ergonomics Association). Definition of Ergonomics. Council of IEA, 2000. Disponivel em: <http://www.iea.cc/01_what/What\%20is\%20Ergonomics. html>. Acesso em 20 abr. 2017.

ISO, 2010. ISO 9241 Part 210: Human-centred design for interactive systems. ISO 9241- 210:2010(E). Genebra: ISO, 2010.

ISO, 1999. ISO 13407: Human-centred Design Processes for Interactive Systems. Switzerland, International Organization for Standardization, Geneva, 1999.

ISO, 2002. ISO/TR 16982: Ergonomics of human-system interaction - Usability methods supporting human-centred design. Switzerland, International Organization for Standardization, Geneva, 2002.

KARWOWSKI, W.; SOARES, M.; STANTON, N. (Ed.) Human Factors and Ergonomics in Consumer Product Design - Methods and Techniques. CRC Press, 2011.

KELLY, J. Displacing use: Exploring alternative relationships in a human-centred design process. Design Studies, v. 35, p. 353-373, 2014.

LEONEL, C. E. L. Sistematização do processo de planejamento da inovação de produtos com enfoque em empresas de pequeno e médio porte. Florianópolis, 2006. Dissertação (mestrado) - Departamento de Engenharia Mecânica, Universidade Federal de Santa Catarina.

MERINO, G. S. A. D. Metodologia para a prática projetual do design: com base no projeto centrado no usuário e com ênfase no design universal. Florianópolis, 2014. Tese (doutorado) - Departamento de Engenharia de Produção, Universidade Federal de Santa Catarina.

MONTANHA JR., I. Sistemática de gestão da tecnologia aplicada no projeto de produtos: um estudo para as empresas metal-mecânicas de micro e pequeno porte. Florianópolis, 2004. Dissertação (mestrado) - Departamento de Engenharia Mecânica, Universidade Federal de Santa Catarina.

MOSSINK, J. C. M. Evaluation of design practice and the implementation of ergonomics. Ergonomics, v.33, n. 5, p. 613-619, 1990. 
NEUMANN, W. P.; VILLAGE, J. Ergonomics action research II: a framework for integrating HF into work system design. Ergonomics, v.55, n.8, p.781-795, 2012.

NIELSEN, J. Usability Engineering. San Diego: Academic Press, 1993.

PHEASANT, S. Bodyspace. Anthropometry, Ergonomics and the Design of Work. London: Taylor \& Francis, 1997.

PREECE, J.; ROGERS, Y.; SHARP, H. Interaction design: Beyond human-computer interaction. New York: John Wiley\& Sons, Inc, 2002.

REINERT, F.; GONTIJO, L. A. O ensino da ergonomia na prática projetual da engenharia. In: 16 Ergodesign, 2017a. Anais do 16 Congresso Internacional de Ergonomia e Usabilidade de Interfaces Humano Tecnológica: Produto, Informações, Ambientes Construídos e Transporte, Florianópolis: Edgar Blucher.

REINERT, F.; GONTIJO, L. A. Análise da abordagem ergonômica nas metodologias de projeto de produto de engenharia. In: 16 Ergodesign, 2017b. Anais do 16 Congresso Internacional de Ergonomia e Usabilidade de Interfaces Humano Tecnológica: Produto, Informações, Ambientes Construídos e Transporte, Florianópolis: Edgar Blucher.

ROMANO, L. Modelo de referência para o processo de desenvolvimento de máquinas agrícolas. Florianópolis, 2003. Tese (Doutorado) - Departamento de Engenharia Mecânica, Universidade Federal de Santa Catarina.

ROZENFELD, H. et al. Gestão de Desenvolvimento de Produtos: Uma referência para a melhoria do processo. São Paulo: Saraiva, 2006.

RUBIN, J., CHISNELL, D. Handbook of Usability Testing: Howto Plan, Design, and Conduct Effective Tests. Indianapolis: Wiley Publishing, 2008.

SAVI, R,; SOUZA, C. B. C. Design centrado no usuário e o projeto de soluções educacionais. E-Tech: Tecnologias para Competitividade Industrial, Florianópolis, n. Especial Design, 2015/1, p 33-52, 2015.

STANTON, N. Human factors is consumer products. London: Taylor \& Francis, 1998.

STANTON, N.; HEDGE, A.; BROOKHUIS, K.; SALAS, E.; HENDRICK, H. The handbook of human factors and ergonomics methods. Boca Raton: CRC Press, 2005.

TULLIS, T.; ALBERT, B. Measuring the User Experience. Amsterdam: Morgan Kauffman, 2008. 